\title{
Controlled hypotensive anesthesia in the beach-chair position under general anesthesia: Is it safe for shoulder arthroscopy?
}

\author{
(1) Mehmet Özgür Özhan'1, (1) Mehmet Burak Eşkin², (1) Ceyda Çaparlar³, (1) Mehmet Anıl Süzer1, (1) Uğur Gönç4, \\ (1) Bülent Atik ${ }^{5}$, (1) Metin Polat ${ }^{4}$
}

${ }^{1}$ Çankaya Hospital, Clinic of Anesthesiology and Reanimation, Ankara, Turkey

2University of Health Sciences Turkey, Gülhane Faculty of Medicine, Department of Anesthesiology and Reanimation, Ankara, Turkey

3University of Health Sciences Turkey, Diskapi Yildirim Beyazit Training and Research Hospital, Clinic of Anesthesiology and Reanimation, Ankara, Turkey

${ }^{4}$ Çankaya Hospital, Clinic of Orthopedics and Traumatology, Ankara, Turkey

${ }^{5}$ Balikesir University Faculty of Medicine, Department of Anesthesiology and Reanimation, Balikesir, Turkey

Date submitted:

16.12.2019

Date accepted:

15.01.2020

Online publication date:

15.06.2020

\section{Corresponding Author:}

Mehmet Burak Eşkin MD, Health

Sciences University Turkey, Gülhane

Faculty of Medicine, Department of

Anesthesiology and Reanimation,

Ankara, Turkey

mehmetburak.eskin@sbu.edu.tr

ORCID:

orcid.org/0000-0001-6781-9334

Keywords: Shoulder arthroscopy, outpatient surgery, beach-chair position, general anesthesia

\begin{abstract}
Aims: The beach-chair position (BCP) imposes a risk to cerebrovascular hypoperfusion due to deep hypotensive events (DHEs) which may progress to catastrophic neurological complications. However, a controlled hypotensive anesthesia (CHA) management is often required for arthroscopic shoulder surgery to reduce blood loss and to improve visibility of surgical field. The aim of this retrospective study was to evaluate CHA managements, DHEs, and complications in patients who underwent shoulder arthroscopy in the BCP under general anesthesia (GA) between years 2017 and 2019.
\end{abstract}

Methods: After hospital's ethic committee approval was obtained, medical records of 72 adult patients were retrospectively reviewed. Primary outcome measures were the frequency of $\mathrm{CHA}$ management and the incidence of DHE. Secondary outcome measures were to determine the stages of surgery at which DHEs have developed and the complications.

Results: CHA was required in 46 of total 72 patients (63.9\%). Among those 46 patients, 31 (67.4\%) had at least one DHE. A total of 82 DHEs were detected in 49 patients whereas mean arterial pressure limits were normal $( \pm 30 \%$ of baseline) in the remaining $23(68.1 \%$ vs. $31.9 \% ; p<0.05)$. DHEs were recorded most frequently after BCP $(p<0.05)$. All DHEs were promptly treated with the discontinuation of $\mathrm{CHA}$ and administration of vasopressor drugs. No neurological complication was observed.

Conclusions: GA in BCP caused DHEs and its incidence was increased by inducing CHA. It was concluded that neurological complications could be prevented when further decrease in blood pressure was avoided or promptly treated in case of a hypotensive event.

\section{Introduction}

Arthroscopy is the most common surgical procedure for the diagnosis and treatment of orthopedic shoulder pathology and generally performed as an outpatient procedure rather than open surgery due to minimal invasive properties and low risk of morbidity (1). Shoulder arthroscopy can be performed under general anesthesia (GA), peripheral nerve block, or a combination of both techniques. Patients are positioned in either beach-chair position (BCP) or lateral decubitus position. The BCP has became more popular than lateral decubitus position because of better visualization of the joint, lower incidence of neurovascular complications, and easier conversion to open surgery (2). Common complications of shoulder arthroscopy are traction injuries in the brachial plexus, extravasation of irrigation 
fluid out of the joint, air embolism, and infection irrespective of positioning and anesthetic management. However, uncommon but devastating complications like stroke, cerebral cord ischemia, and transient visual loss have been reported for the BCP. The exact mechanisms of these complications are not clear, but studies have reported that intraoperative deep hypotension events may cause cerebral hypoperfusion which is defined as a reduction in mean arterial blood pressure (BP) below $50 \mathrm{mmHg}$. It is reported that deep hypotensive events may arise from upright positioning combined with GA (3-5). For this reason, it is advocated that the mean arterial BP should be kept in a range between 50 and $65 \mathrm{mmHg}$ during shoulder arthroscopy, which does not blunt cerebral blood flow and autoregulation. However, a controlled hypotensive anesthesia ( $\mathrm{CHA}$ ) technique is often required to reduce blood loss and to improve visibility of surgical field during arthroscopy which may further increase cerebral hypoperfusion (4).

The aim of this retrospective study was to evaluate the $\mathrm{CHA}$ management, deep hypotensive events, and complications in patients who underwent shoulder arthroscopy in the BCP under GA between years 2016 and 2018. Primary outcome measures were the frequency of CHA management and the incidence of deep hypotension events. Secondary outcome measures were to determine at which stages of the surgery deep hypotension events developed and perioperative complications.

\section{Methods}

Approval from the Local Research Ethics Committee of our tertiary hospital was obtained before initiating the study (University of Medical Sciences Turkey, Gülhane Training and Research Hospital, project no: 18/102, date: 02.05.2018). After obtaining hospital ethic committee approval, medical files and anesthesia charts of the patients who underwent arthroscopic surgery were retrospectively evaluated. Inclusion criteria were unilateral elective shoulder surgery, arthroscopic procedure, and GA in the BCP. Exclusion criteria were urgent surgery, arthroscopic surgery converted to open surgery, BCP converted to lateral decubitus position, patients with history of cerebrovascular disease or neurological injury, and missing data.

\section{Anesthetic management}

GA was induced using intravenous (IV) propofol, fentanyl, and rocuronium. Propofol infusion or sevoflurane inhalation combined with IV remifentanil was used for the maintenance. $\mathrm{CHA}$ was induced in patients with a normal range of mean arterial pressure (MAP); i.e. $\pm 20 \%$ of baseline MAP after surgical team stated that the quality of surgical exposure was not adequate due to bleeding. IV metoprolol or nitroglycerine was given to achieve $\mathrm{CHA}$ defined as a reduction in the MAP to a range of $60-70 \mathrm{mmHg}$ or $\pm 30 \%$ of baseline levels. Non-invasive BP measurement was used for American Society of Anesthesiologists (ASA) I patients while invasive BP measurement was used for the remaining patients. A deep hypotension event was defined when the MAP reduced to $<50 \mathrm{mmHg}$ or systolic BP to $<90 \mathrm{mgHg}$. Deep hypotensive events were treated with the discontinuation of $\mathrm{CHA}$, reducing of anesthetic drug doses, IV bolus fluid replacement, and administration of 5-10 mg IV ephedrine boluses. Patients were evaluated using the modified Aldrete Scoring System (mASS) after GA in the post-anesthesia care unit, and patients with a mASS score of $>9$ were transferred to the service.

Following data were collected and evaluated from patient's files and anesthesia charts: a) demographic data: Gender, age, body height, weight, ASA physical status, and co-morbidity, b) duration of surgery (min.), c) anesthetic management, d) number of CHA management, e) number, duration, and stage of deep hypotensive events, f) perioperative complications, g) time to discharge (hours).

\section{Statistical Analysis}

Statistical analysis was performed using IBM SPSS Statistics version 21 (IBM SPSS Inc., Chicago, IL). Descriptive statistics were used as mean and standard deviation (Mean $\pm \mathrm{SD}$ ) for continuous data, and frequency and percentage (n, \%) for categorical data. The normal distribution of the continuous data was evaluated with the Kolmogorov-Smirnov test. The abnormal distribution for continuous variables was analyzed using the Mann-Whitney $U$ test between the groups whereas the Pearson chi-square test was employed for categorical variables. $\mathrm{P}<0.05$ was considered as statistically significant.

\section{Results}

Records of 96 patients were evaluated, and 24 of those patients were excluded due to missing data (Figure 1). Demographic data of the remaining 72 patients were shown in Table 1 . The mean age was $49.84 \pm 15.25$ years and co-morbidity rate was $50 \%$ as follows: Hypertension $(20.8 \%)$, coronary artery disease $(9.7 \%)$, diabetes mellitus $(9.7 \%)$, coronary artery disease + diabetes mellitus ( $4.2 \%)$, and hypertension + diabetes mellitus (5.6\%).

Primary outcome measures: A minimum one episode of deep hypotensive event was recorded in 49 patients whereas the MAP levels were remained in normal range in the remaining 23 patients [ $49(68.1 \%)$ vs. $23(31.9 \%)$; $p<0.05$ ]. It was observed that $\mathrm{CHA}$ was required in 46 patients and not required in 26 patients $(63.9 \%$ vs. $36.1 \%$; $p<0.05)$. Among 46 patients who received $\mathrm{CHA}$, deep hypotensive event was recorded in 31 patients while arterial BP remained in normal range in other 15 patients $(67.4 \%$ vs. $32.6 \%$; $p<0.05)$. On the other hand, a deep hypotensive event was recorded in 18 patients out of 26 patients who did not receive CHA [18 (69.2\%) vs. $8(30.8 \%)$; $p<0.05]$. The rate of deep hypotensive event was statistically insignificant 
between patients who did receive CHA or not $(67.4 \%$ vs. $69.2 \%$; $p>0.05)$. The total number of deep hypotensive events was 82 . Primary outcome measures were summarized in Table 2.

Secondary outcome measures: It was observed that deep hypotensive events developed most often after the BCP

\begin{tabular}{|c|c|c|}
\hline Parameter & & Result \\
\hline \multirow{2}{*}{ Gender (n, \%) } & Female & $36(50 \%)$ \\
\hline & Male & $36(50 \%)$ \\
\hline \multicolumn{2}{|l|}{ Age (yrs) } & $49.8 \pm 15.2$ \\
\hline \multicolumn{2}{|l|}{ Height (cm) } & $167.0 \pm 10.8$ \\
\hline \multicolumn{2}{|l|}{ Weight (kg) } & $79.5 \pm 13.3$ \\
\hline \multirow{3}{*}{ ASA physical status } & 1 & $36(50.0 \%)$ \\
\hline & II & $28(38.9 \%)$ \\
\hline & III & $8(11.1 \%)$ \\
\hline \multirow{6}{*}{ Co-morbidity } & None & $36(50.0 \%)$ \\
\hline & HT & $15(20.8 \%)$ \\
\hline & CAD & $7(9.7 \%)$ \\
\hline & $\mathrm{DM}$ & $7(9.7 \%)$ \\
\hline & $C A D+C V D$ & $3(4.2 \%)$ \\
\hline & $\mathrm{HT}+\mathrm{DM}$ & $4(5.6 \%)$ \\
\hline \multicolumn{2}{|l|}{ Operation time (min.) } & $97.8 \pm 20.1$ \\
\hline \multicolumn{2}{|l|}{ Discharge time (hrs) } & $20.8 \pm 4.7$ \\
\hline \multicolumn{3}{|c|}{$\begin{array}{l}\text { ASA: American Society of Anesthesiologists, HT: Hypertension, CAD: } \\
\text { Coronary artery disease, DM: Diabetes mellitus, CVD: Cerebrovascular } \\
\text { disease, Yrs: Years, Hrs: Hours, Min.: Minimum }\end{array}$} \\
\hline
\end{tabular}

Table 2. Controlled hypotensive anesthesia and deep hypotensive events

\begin{tabular}{|c|c|c|c|}
\hline Parameter & & n (\%) & $p^{*}$ \\
\hline \multirow{2}{*}{$\begin{array}{l}\text { Patients } \\
\text { received } \mathrm{CHA} \\
(\mathrm{n}, \%)\end{array}$} & Yes & $46(63.9 \%)$ & \multirow{2}{*}{$<0.05$} \\
\hline & No & $26(36.1 \%)$ & \\
\hline \multirow{2}{*}{$\begin{array}{l}\text { Patients with } \\
\mathrm{DHE}(\mathrm{n}, \%)\end{array}$} & 0 episode & $23(31.9 \%)$ & \multirow{2}{*}{$<0.05$} \\
\hline & $\geq 1$ episode & $49(68.1 \%)$ & \\
\hline \multirow{2}{*}{$\begin{array}{l}\text { Patients with } \\
\text { DHE }(n, \%)\end{array}$} & Received CHA & $31(67.4 \%)$ & \multirow[b]{2}{*}{$>0.05$} \\
\hline & $\begin{array}{l}\text { Not received } \\
\mathrm{CHA}\end{array}$ & $18(69.2 \%)$ & \\
\hline \multirow{3}{*}{$\begin{array}{l}\text { Number of DHE } \\
\text { (total } 82 \text { ) }\end{array}$} & 1 & $21(42.9 \%)$ & \multirow{3}{*}{$>0.05$} \\
\hline & 2 & $23(46.9 \%)$ & \\
\hline & 3 & $5(10.2 \%)$ & \\
\hline \multirow{5}{*}{$\begin{array}{l}\text { Stage of } \\
\text { DHE in the } \\
\text { perioperative } \\
\text { period }\end{array}$} & After induction & $12(14.6 \%)$ & \multirow{5}{*}{$<0.05$} \\
\hline & After positioning & $39(47.6 \%)$ & \\
\hline & During surgery & $30(36.6 \%)$ & \\
\hline & After extubation & $1(1.2 \%)$ & \\
\hline & In the ward & $0(0.0 \%)$ & \\
\hline \multicolumn{4}{|c|}{$\begin{array}{l}\text { * } p<0.05 \text { was considered as statistically significant. } \\
\text { MAP: Mean arterial pressure, CHA: Controlled hypotensive anesthesia, DHE } \\
\text { Deep hypotensive event (MAP } \leq 50 \mathrm{mmHg} \text { ) }\end{array}$} \\
\hline
\end{tabular}

of patients followed by intraoperative period, in other words, after the start of CHA ( $p<0.05$; Table 2). When the anesthesia records on the chart were evaluated, it was found that the duration of deep hypotensive events did not exceed 5 minutes. A total of 9 complications, which included 7 postoperative nausea and vomiting and 2 bronchospasms, were recorded in 7 of 72 patients. No neurological complications were observed.

\section{Discussion}

The results of this study showed that $\mathrm{CHA}$ was required in most of patients who underwent arthroscopic shoulder surgery. The incidence of deep hypotensive events was found to be mainly increased with the BCP combined with GA and further precipitated with the administration of $\mathrm{CHA}$, but complications related to cerebral hypoperfusion were not observed throughout the procedure. Interventions to prevent further decreases in BP might preserve patient's neurological status.

As in other various surgeries, a clear and bloodless surgical exposure is important in arthroscopic shoulder surgery. It was reported that a pressure difference should be ensured between systolic BP and pressure in the subacromial space to produce a bloodless surgical field. Increasing the arthroscopic pump pressure, decreasing the systolic BP or a combination of both are generally used for this purpose. However, high irrigation pump pressure may cause excessive extra-articular fluid extravasation, which may progress to serious complications including neurovascular impingement and tracheal stenosis. So, a combination of both techniques (low pump pressure and induced hypotension) is much more preferred (6).

Intraoperative induced hypotension is known to reduce perioperative blood loss and duration of the surgery up to $50 \%$ but may cause serious complications by decreasing perfusion of vital organs (4). Especially, neurological complications related to cerebral hypoperfusion may have a potential to progress to brain and spinal cord injury (5). Although the incidence of this complication is quite low (8 in 224275 patients) during shoulder arthroscopy, it is remarkable that all events were observed in the BCP under GA (3). Upright positioning of an awake patient activates sympathetic nervous system and thereby increases peripheral vascular resistance, which results in elevation of arterial BP. However, GA blinds baroreceptor responses which is required to correct of the effects of the gravity on the cerebral perfusion pressure. In addition, GA also prevents peripheral vascular resistance. In our study, it was found that nearly half of all deep hypotensive events were developed after positioning of patients. It was reported that the rate of intraoperative hypotension could be as high as $32 \%$ during GA in upright position (7). In our study, the rate of hypotensive events was similar between the patients who received or not received $\mathrm{CHA}$. According to this, it may be conceivable that the BCP combined 
with GA had an great effect on deep hypotensive events without the administration of $\mathrm{CHA}$.

CHA has been used for more than 60 years in anesthesia, but there are still controversies about the safe limits of the management (8). The common opinion is that the MAP should be kept above $60 \mathrm{mmHg}$, systolic BP above 90-100 $\mathrm{mmHg}$ while maximum reduction in MAP should not exceed $30 \%$ of basal levels (9). However, many studies revealed that regional cerebral desaturation episodes were diagnosed using near-infrared spectroscopy in about $80 \%$ of patients even intraoperative MAP was kept between $\pm \% 20$ of baseline levels. However, any neurological complication was not reported $(9,10)$.

In our study, minimum one deep hypotensive episode was recorded in $68.1 \%$ of patients. Further decreases in MAP were prevented with interventions including the discontinuation of anesthetic and hypotensive agents and administration of ephedrine and fluid boluses. In a study by Gillespie et al. (11), a minimum one episode of a decrease in systolic arterial pressure below $90 \mathrm{mmHg}$ was reported in all patients and the mean rates of decrease in systolic and mean arterial BP were $36 \%$ and $42 \%$, respectively. Among these patients, an ischemic change was detected with electroencephalography only in 3 patients without any neurological impairment. Therefore, it was advocated that the risk of neurological complications may have a relationship with the severity of the deep hypotensive events as well as with its duration. In our study, although the exact duration of deep hypotensive event was not strictly recorded during the operation, anesthesia records revealed that any of deep hypotensive event did not exceed five minutes.

Another debate continues on where the cuff of noninvasive BP or transducer of invasive arterial pressure line places to correctly measure the BP at brain level. It is not exactly established whether a non-invasive BP measurement reflects cerebral BP. Also, the question concerns whether the transducer should be adjusted according to the level of the heart or of theear (external auditor meatus) (12). One opinion is that the measurement with non-invasive BP should be corrected according to the position of the patient's head (a reduction in BP of $1 \mathrm{mmHg}$ for each $1.25 \mathrm{~cm}$ height difference between the site of measurement and the brain) $(9,13)$. In our daily anesthetic practice, the level of invasive pressure transducer or of noninvasive pressure cuff is adjusted to the level of the heart but corrected to the level of the external auditor meatus to measure cerebral perfusion pressure.

Our study has some limitations. Firstly, there was a lack of measurement of cerebral perfusion using electroencephalogram or near-infrared spectroscopy. Both are advanced and expensive techniques and need experience, which limit their use in daily practice. The second limitation is that the nature of the retrospective design of the study may cause selection errors and comparison bias. In order to prevent this, we tried to collect all data of patients and excluded 24 files with missing data (Figure 1).

\section{Conclusion}

As a result, it is concluded that the combination of the BCP with GA caused deep hypotensive events which were further precipitated by inducing CHA during shoulder arthroscopy. We think that neurological complications could be prevented when further decreases in BP would be avoided or promptly treated in case of a hypotensive event.

\section{Ethics}

Ethics Committee Approval: Approval of the Local Research Ethics Committee of our tertiary hospital was obtained before initiating the study (University of Medical Sciences Turkey, Gülhane Training and Research Hospital, project no: 18/102, date: 02.05.2018).

Informed Consent: Retrospective study.

Peer-review: Externally peer-reviewed.

\section{Authorship Contributions}

Surgical and Medical Practices: M.Ö.Ö., C.Ç., Concept: M.Ö.Ö., M.B.E., U.G., Design: M.Ö.Ö., M.B.E., B.A., Data Collection or Processing: M.Ö.Ö., M.B.E., M.P., Analysis or Interpretation: M.B.E., C.Ç., M.A.S., M.P., Literature Search: M.B.E., C.Ç., B.A., Writing: M.Ö.Ö., M.B.E., U.G., B.A., M.P.

Conflict of Interest: No conflict of interest was declared by the authors.

Financial Disclosure: The authors declared that this study received no financial support.

\section{References}

1. Jain NB, Higgins LD, Losina E, Collins J, Blazar PE, Katz JN. Epidemiology of musculoskeletal upper extremity ambulatory surgery in the United States. BMC Musculoskelet Disord. 2014;15:24.

2. Paxton ES, Backus J, Keener J, Brophy RH. Shoulder arthroscopy: basic principles of positioning, anesthesia, and portal anatomy. J Am Acad Orthop Surg. 2013;21:332342.

3. Friedman DJ, Parnes NZ, Zimmer Z, Higgins LD, Warner JJ. Prevalence of cerebrovascular events during shoulder surgery and association with patient position. Orthopedics. 2009:32.

4. Kotha R, Orebaugh SL. Shoulder Surgery in the Beach Chair Position. Adv Anesth. 2014;32:37-57.

5. Pohl A, Cullen DJ. Cerebral ischemia during shoulder surgery in the upright position: a case series. J Clin Anesth. 2005;17:463-469. 
6. Rains DD, Rooke GA, Wahl CJ. Pathomechanisms and Complications Related to Patient Positioning and Anesthesia During Shoulder Arthroscopy Arthroscopy. Arthroscopy. 2011;27:532-541.

7. Murphy GS, Szokol JW. Blood pressure management during beach chair position shoulder surgery: What do we know? Can J Anesth. 2011;58:977-982.

8. Hampton LJ, Little DM Jr. Complications associated with the use of controlled hypotension in anesthesia. AMA Arch Surg. 1953;67:549-556.

9. Murphy GS, Szokol JW, Marymont JH, et al. Cerebral oxygen desaturation events assessedby near-infrared spectroscopy during shoulder arthroscopy in the beach chair and lateral decubitus positions. Anesth Analg. 2010;111:496-505.
10. Lee JH, Min KT, Chun YM, Kim EJ, Choi SH. Effects of beach-chair position and induced hypotension on cerebral oxygensaturation in patients undergoing arthroscopic shoulder surgery. Arthroscopy. 2011;27:889-894.

11. Gillespie R, Shishani Y, Streit J, etal. The Safety of Controlled Hypotension for Shoulder Arthroscopy in the Beach-Chair Position. J Bone Joint Surg Am. 2012;94:1284-1290.

12. Drummond JC, Hargens AR, Patel PM. Hydrostatic gradient is important:blood pressure should be corrected (letter). APSF Newsletter. 2009;24:6.

13. Laflam A, Joshi B, Brady K, et al. Shoulder surgery in the beach chair position is associated with diminished cerebral autoregulation but no differences in postoperative cognition or brain injury biomarker levels compared with supine positioning: the anesthesia patient safety foundation beach chair study. Anesth Analg. 2015;120:176-185. 